\title{
An evaluation of heuristics for scheduling a non-delay permutation flow shop with family setups to minimize total earliness and tardiness
}

\author{
J Schaller $^{* 1}$ and JMS Valente ${ }^{2}$ \\ ${ }^{1}$ Eastern Connecticut State University, Willimantic, CT, USA; and ${ }^{2}$ Universidade do Porto, Porto, \\ Portugal
}

This paper presents several procedures for developing non-delay schedules for a permutation flow shop with family setups when the objective is to minimize total earliness and tardiness. These procedures consist of heuristics that were found to be effective for minimizing total tardiness in flow shops without family setups, modified to consider family setups and the total earliness and tardiness objective. These procedures are tested on several problem sets with varying conditions. The results show that variable greedy algorithms are effective when solving small problems, but using a genetic algorithm that includes a neighbourhood defined by the sequence of batches of jobs belonging to the same set-up family is effective when solving medium- or large-sized problems. The results also show that if setup times can be reduced a significant reduction in total earliness and tardiness could result.

Journal of the Operational Research Society (2013) 64, 805-816. doi:10.1057/jors.2012.94

Published online 22 August 2012

Keywords: scheduling; flow shop; heuristics; family setups

\section{Introduction}

In many operations obtaining economies of scale is a critical element in achieving success. Economies of scale are efficiencies in production in which per-unit production costs increase at a slower rate than production volume. In scheduling, efficiencies that lead to economies of scale are gained by grouping similar jobs together. The motivation for grouping sometimes relates to change-over times, or setup times, on the machines. For example, jobs may belong to families where the jobs in each family tend to be similar in some way, such as their required tooling. As a result of this similarity, a job does not need a setup when following another job from the same family, but a known 'family setup time' is required when a job follows a member of some other family. Typically, there are a large number of jobs, but a relatively small number of families.

The widespread adoption of lean production methods has caused customers to view early delivery of products as well as tardy delivery to be undesirable. Early deliveries result in unnecessary inventory that ties up cash as well as space and resources needed to maintain and manage inventory. Therefore, an important consideration when

\footnotetext{
${ }^{*}$ Correspondence: $J$ Schaller, Department of Business Administration, Eastern Connecticut State University, 83 Windham St., Willimantic, CT 06226-2295, USA.
}

sequencing and scheduling a set of jobs is completing each job on the customer's due date.

To address these considerations, this paper seeks to identify and compare methods for sequencing a set of jobs in a permutation flow shop with significant family setup times that will minimize the total earliness and tardiness of the jobs. Most research on flow shops has assumed the sequence of jobs to process will be the same on each machine. These schedules are referred to as permutation schedules. This is done for two reasons. First, it simplifies the computational effort and second, it is often not practical to change the sequence of jobs from one machine to the next. In this research only permutation schedules are considered.

Formally, suppose there is a set of $n$ jobs belonging to $F$ setup families to be processed in a flowshop consisting of $M$ machines. Let $f_{j}$ and $d_{j}$ represent the setup family and the due date of job $j(j=1, \ldots, n)$ respectively. Let $p_{j m}, S_{j m}$, and $C_{j m}$ represent the processing time, setup time, and completion time of job $j(j=1, \ldots, n)$ on machine $m$ $(m=1, \ldots, M)$, respectively. The earliness of job $j, E_{j}$, is defined as: $E_{j}=\max \left\{d_{j}-C_{j M}, 0\right\}$, for $j=1, \ldots, n$ and the tardiness of job $j, T_{j}$, is defined as: $T_{j}=\max \left\{C_{j M}-d_{j}, 0\right\}$, for $j=1, \ldots, n$. The objective function, $Z$, can be expressed as: $Z=\sum_{j=1}^{n} E j+T_{j}$.

Since the objective in the problem is non-regular, inserting idle time into a schedule for the jobs can help to reduce the earliness of some jobs and thus improve the objective. 
However, in certain production environments, the insertion of idle time may actually be undesirable, or even impossible. For instance, idle time should be avoided when the capacity of the shop is limited when compared with the demand. Also, idle time should not be inserted when the machine has a high operating cost, and/or when starting a new production run involves high setup costs or times. Some specific examples of production environments where the insertion of idle time is undesirable have been given by Korman (1994) and Landis (1993). In this research only non-delay schedules without unforced inserted idle time are considered. Also, setups for a machine can be performed in anticipation of a job arriving at a machine; therefore if the job to be sequenced in position $j$ is denoted as $[j]$ and $C_{[0] 1}=0$, then $C_{[j] 1}=C_{[j-1] 1}+p_{[j] 1}$ if $f_{[j]}=f_{[j-1]}$ and $C_{[j] 1}=\mathrm{C}_{[j-1] 1}+S_{[j] 1}+p_{[j] 1}$ if $f_{[j]} \neq f_{[j-1]}$, and $C_{[j] m}=\max$ $\left\{C_{[j] m-1}, C_{[j-1] m}\right\}+p_{[j] m}$ if $f_{[j]}=f_{[j-1]}$ and $\max \left\{C_{[j] m-1}\right.$, $\left.C_{[j-1] m}+S_{[j m]}\right\}+p_{[j] m}$ if $f_{[j]} \neq f_{[j-1]}$ for $m=2, \ldots, M$.

Streams of literature that are most relevant to the problem addressed in this paper are minimizing total earliness and tardiness in a flow shop and scheduling flow shops with family setup times. A large number of papers have been published on scheduling models with earliness and tardiness costs. Baker and Scudder (1990) provide an excellent survey of the initial work on early/tardy scheduling. A recent survey of multi-criteria scheduling which includes problems with earliness and tardiness penalties is given in Hoogeveen (2005). Most of the research with an objective based on early and tardy job completion costs deals with single machine environments. Recent research for single machine environments with an early/tardy objective and no idle time is summarized in Valente (2009). Scheduling models with inserted idle time, on the other hand, are reviewed in Kanet and Sridharan (2000).

Only three papers address objectives based on earliness and tardiness costs in flow shops. Moslehi et al (2009) present an optimal procedure for minimizing the sum of maximum earliness and tardiness in a two-machine flow shop. Chandra et al (2009) present approaches for permutation flow shop scheduling with earliness and tardiness penalties when all the jobs have a common due date. Madhushini et al (2009) present branch-and-bound algorithms for scheduling permutation flow shops for a variety of objectives including minimizing earliness and tardiness without inserted idle time.

An objective that is partially related to the objective considered in this paper is minimizing total tardiness. The reason the objectives are related is that our objective includes total tardiness. Vallada et al (2008) reviewed and tested over 40 heuristic methods for the m-machine flow shop problem with the objective of minimizing total tardiness. Based on their tests, Vallada et al (2008) found that the neighbourhood searches developed by Kim et al (1996) were the best heuristics and the simulated annealing algorithms developed by Parthasarathy and Rajendran
(1997) and Hasija and Rajendran (2004) were the best meta-heuristics. Framinan and Leisten (2008) developed a variable greedy algorithm for the problem and found it to be more effective in minimizing total tardiness than the simulated annealing algorithms developed by Parthasarathy and Rajendran (1997) and Hasija and Rajendran (2004). Vallada and Ruiz (2010) proposed three genetic algorithms for minimizing total tardiness in permutation flow shops and performed computational tests for these algorithms and other metaheuristics including those by Parthasarathy and Rajendran (1997) and Hasija and Rajendran (2004). They found that two of the genetic algorithms performed the best.

Cheng et al (2000) provide a review of flow shop scheduling research with setup times. Most of the papers identified in this review consider the objective of minimizing makespan. The only paper that considered an objective that included total earliness and tardiness was Jorden (1996). In this paper a genetic algorithm was developed for a two-machine flow shop with family setup times to minimize the sum of weighted earliness and tardiness penalties.

Section 2 describes the proposed scheduling procedures. Section 3 presents the computational tests of the procedures as well as the results of the tests. Section 4 describes a test to measure the effect of a reduction in setup times and presents the results. Section 5 concludes the paper.

\section{Heuristic procedures}

Six heuristic procedures that are based on procedures that were found to be effective for minimizing total tardiness in flow shops without family setups are described in this section. The first two procedures are neighbourhood search procedures. Two procedures are variable greedy algorithms and two are genetic algorithms.

\subsection{Neighbourhood search procedures}

Kim et al (1996) developed two neighbourhood searches for minimizing total tardiness in flow shops without family setups. One of the searches used an exchange operator to define the neighbourhood and the other used an insert operator. Two neighbourhood search procedures are presented in this section. The first uses both of the neighbourhood operators developed by Kim et al (1996). This procedure is referred to as NS in this paper. The second uses the exchange and insert operators on individual jobs and also uses the exchange and insert operators as well as a consolidate operator on batches of jobs to define neighbourhoods. This procedure is referred to as BNS in this paper.

In both procedures an initial sequence is developed and then the sequence is improved by using the neighbourhood search operators. The neighbourhood search operators are repeated until none of the moves in a neighbourhood offer an improvement. The algorithms both use the same 
procedure to find an initial sequence. The initial sequence is found using a method that is similar to that used in Kim et al (1996) with a modification to the objective. This procedure is referred to as the IS procedure.

2.1.1. Initial sequence (IS procedure). The initial sequence is created in two steps. First, a list of the jobs is created. Then, an insertion algorithm is used to create a sequence. To create a list of the jobs they are first sorted in non-decreasing order of due dates. The insertion algorithm starts by selecting the first two jobs on the list and two sequences are formed consisting of these jobs (each job is placed first once and second once). The total earliness and tardiness is calculated for each of these sequences and the sequence with the lower total earliness and tardiness is selected as the initial sequence. The insertion step is then repeated $n-2$ times to sequence the remaining jobs. Let $k=3$ be the first time the insertion step is performed. The insertion step is performed as follows. Pick the $k$ th job from the initial list and create $k$ sequences by inserting this job into each possible position of the sequence while keeping the relative order of the jobs from the $k$-1st iteration. The total earliness and tardiness is then calculated for each of these new sequences. The sequence with the lowest total earliness and tardiness is selected. If $k<n$, then let $k=k+1$ and the insertion step is repeated with the sequence just selected as the initial sequence. If $k=n$ the phase stops.

The initial list can be created in $\mathrm{O}(n \ln (n))$ time. The number of insertions evaluated is on the order of $\mathrm{O}\left(n^{2}\right)$ and each partial sequence can be evaluated in $\mathrm{O}(n)$ time so the complexity of the procedure is $\mathrm{O}\left(n^{3}\right)$.

\subsubsection{NS neighbourhood search improvement proce-} dure. The NS neighbourhood search improvement procedure attempts to improve a sequence by performing neighbourhood searches. The two neighbourhood searches used in Kim et al (1996) are performed. The first search looks at all of the possible exchanges of pairs of jobs. A sequence of jobs $\sigma$ is converted into a sequence $\sigma$ by exchanging the positions of two jobs $j$ and $k$. If the total earliness and tardiness of sequence $\sigma$ is less than the total earliness and tardiness of the best sequence found so far (incumbent sequence), then the incumbent is updated. The second search is an insertion search. Each job is removed from a sequence $\sigma$ and then $n$ sequences are created by inserting the job into each possible position while maintaining the relative order of the other jobs. If the total earliness and tardiness of a sequence is less than the total earliness and tardiness of the incumbent sequence, then the incumbent is updated.

The two searches are repeated until either of two conditions occurs: (1) both searches fail to obtain an improvement or (2) a time limit is exceeded. The time limit is set to $n \times(\mathrm{M} / 2) \times 0.125 \mathrm{~s}$. This time limit was based on preliminary computational experiments and was set for all of the procedures described in this section.

To evaluate a neighbourhood using the exchange (insertion) search $\mathrm{O}\left(n^{2}\right)$ exchanges (insertions) are needed. Since each sequence can be evaluated in $\mathrm{O}(n)$ time, the complexity of the procedure is $\mathrm{O}\left(n^{3}\right)$ each time a neighbourhood is evaluated.

\subsubsection{BNS neighbourhood search improvement pro-} cedure. The BNS neighbourhood search improvement procedure also attempts to improve a sequence by performing neighbourhood searches. Five neighbourhood searches are performed in this procedure. The first two searches are the job exchange and insertion searches used in Kim et al (1996) and in the NS procedure described in the previous subsection. The other three searches use search operators that explore the neighbourhood of a sequence based on the batches of jobs in the sequence. A batch of jobs is defined as a succession of jobs in a sequence that belongs to the same setup family. Let $\beta$ be a sequence of batches and TET $(\beta)$ be the resulting total earliness and tardiness of sequence $\beta$. Let $n_{\beta}$ be the number of batches in the sequence $\beta$. Three operators are used to explore the neighbourhood of sequence $\beta$ : Consol, BE and BI.

The Consol operator consolidates batches to eliminate setups. A sequence $\beta$ is formed using this operator by combining two batches in $\beta$ and inserting the combined batch into a position of a batch sequence that maintains the relative order of the non-combined batches of $\beta$. Each possible position for the combined batch is checked. If the best of the neighbourhood batch sequences results in a lower total earliness and tardiness than the batch sequence $\beta$, then the best batch sequence is retained. The Consol operator is repeated until it fails to find a better batch sequence.

The $\mathrm{BE}$ operator attempts to improve a sequence by exchanging pairs of batches. A sequence $\beta$ is converted to a sequence of batches $\beta$ by exchanging the positions of two batches $b 1$ and $b 2$. If the total earliness and tardiness of the sequence resulting from the change is less than the total earliness and tardiness of the original sequence, the exchange is retained; otherwise the exchange is reversed.

The BI operator is similar to the job insertion operator. Each batch is removed from the sequence $\beta$ and then $n_{\beta}$ batch sequences are created by inserting the batch into each possible position while maintaining the relative order of the other batches. If the best of the neighbourhood batch sequences results in a lower total earliness and tardiness than the batch sequence $\beta$, then the best batch sequence is retained.

A small example with eight jobs and two families is used to demonstrate how the three batch operators create 
Table 1 Example 1 data

\begin{tabular}{llllllllr}
\hline Job & 1 & 2 & 3 & 4 & 5 & 6 & 7 & 8 \\
\hline Family & 1 & 1 & 2 & 1 & 2 & 2 & 1 & 2 \\
\hline
\end{tabular}

neighbourhoods. Table 1 shows the setup family for each job.

If the current sequence is: $5-3-7-1-8-6-4-2$, then there are four batches consisting of: 5-3 (b1), 7-1 (b2), 8-6 (b3) and 4-2 (b4). If the consol operator is to be performed on batches 2 and 4 (b2 and b4), a new batch is formed 7-1-4-2 and the following three sequences are checked.

$7-1-4-2-5-3-8-6$

$5-3-7-1-4-2-8-6$

$5-3-8-6-7-1-4-2$

The lowest total earliness and tardiness among the sequences above is compared with the total earliness and tardiness of the current sequence and if it is lower the current sequence is updated.

If the BE operator is to be performed on batches 2 and 4 , then the sequence 5-3-4-2-8-6-7-1 is created and checked to see if its total earliness and tardiness is less than the current sequence's and if so the current sequence is updated.

If the BI operator is to be performed on batch 2 (b2), then the following sequences are created.

$7-1-5-3-8-6-4-2$

$5-3-7-1-8-6-4-2$

$5-3-8-6-7-1-4-2$

$5-3-8-6-4-2-7-1$

The lowest total earliness and tardiness among the sequences above is compared with the total earliness and tardiness of the current sequence and if it is lower the current sequence is updated.

The five operators are repeated until either of two conditions occurs: (1) all five operators fail to obtain an improvement or (2) a time limit is exceeded.

Each time a neighbourhood is searched the exchange and insertion searches require $\mathrm{O}\left(n^{3}\right)$ time (see the analysis of the NS procedure). The BE and BI operators require $\mathrm{O}\left(n_{\beta}^{2}\right)$ exchanges and insertions respectively, and each sequence requires $\mathrm{O}(n)$ time to evaluate; therefore, these operators require $\mathrm{O}\left(n^{3}\right)$ time. The consol operator evaluates $\mathrm{O}\left(n_{\beta}^{3}\right)$ sequences with each sequence requiring $\mathrm{O}(n)$ time to evaluate, so this operator requires $\mathrm{O}\left(n^{4}\right)$ time. Therefore the complexity of this procedure, each time a neighbourhood is searched, is $\mathrm{O}\left(n^{4}\right)$.

\subsection{Variable greedy algorithm based on Framinan and Leisten's (2008) variable greedy algorithm}

A variable greedy algorithm combines features of both the variable neighbourhood search (VNS) and the iterated greedy (IG) algorithm meta-heuristics. An IG algorithm generates solutions by using a destruction phase and a construction phase on a constructive heuristic. During the destruction phase $k$ elements are removed from a solution to form a partial solution. During the construction phase a greedy constructive heuristic obtains a potential new solution by adding the previously removed elements. A VNS is used to overcome the problem of the IG stalling in a local optimum. When a VNS is used the size of the neighbourhood that will be searched varies or, in the case of combining VNS with IG, the number of elements $k$ that will be removed and then added back varies.

Let $\sigma$ be a sequence and $C_{j}(\sigma)$ the completion time of job $j$ in the sequence $\sigma$. Define $e t_{j}(\sigma)$ as the slack of job $j$ in the sequence $\sigma$, with $e t_{j}(\sigma)=\max \left\{d_{j}-C_{j}(\sigma), C_{j}(\sigma)-d_{j}\right\}$. The following early/tardy-based greedy algorithm, which is based on Framinan and Leisten (2008)'s slack-based greedy algorithm, is used to obtain a sequence $\sigma^{\prime}$ from another sequence $\sigma$ :

1. Remove from $\sigma$ the $k$ jobs with the largest $e t_{j}(\sigma)$. Sort them in non-increasing order of $e t_{j}(\sigma)$ and store them in $R_{k}$. Let $\mu$ be the remaining subsequence after removing the $k$ jobs.

2. For each job $r$ belonging to $R_{k}$, repeat:

(a) Insert job $r$ in all possible positions of $\mu$. Let $v$ be the best subsequence obtained, and $b$ the position of job $r$ in $v$.

(b) Perform an adjacent pairwise exchange among all jobs in positions $b+1$ to $(n-k+1)$. Let $\pi$ be the best sequence obtained by this approach. If $\pi$ is better than $v$, then $\pi$ becomes $\mu$; otherwise $v$ becomes $\mu$.

\section{Set $\sigma$ equal to $\mu$.}

A small example with eight jobs is used to demonstrate the early/tardy-based greedy algorithm with $k=2$. Table 2 shows the sequence $\sigma$ and the resulting earliness or tardiness of each job in the sequence.

Since $k=2 R_{2}=\{2,6\}$ and the initial subsequence is $5-4-1-7-8-3$. Job 2 is inserted into each possible position of the subsequence creating the following subsequences: $2-5-4-1-7-8-3,5-2-4-1-7-8-3,5-4-2-1-7-8-3$, 5-4-1-2-7-8-3, 5-4-1-7-2-8-3, 5-4-1-7-8-2-3, and 5-41-7-8-3-2. The subsequence $5-4-1-7-8-3-2$ had the lowest total earliness and tardiness so then job 6 is

Table 2 Example 2 data

\begin{tabular}{lcccccccc}
\hline Sequence & 2 & 5 & 6 & 4 & 1 & 7 & 8 & 3 \\
\hline Early/tardy & 862 & 618 & 626 & 551 & 115 & 61 & 304 & 485 \\
\hline
\end{tabular}


inserted into each possible position of this subsequence creating the following sequences: $6-5-4-1-7-8-3-2,5-6-$ 4-1-7-8-3-2, 5-4-6-1-7-8-3-2, 5-4-1-6-7-8-3-2, 5-41-7-6-8-3-2, 5-4-1-7-8-6-3-2, 5-4-1-7-8-3-6-2, and $5-4-1-7-8-3-2-6$. The sequence 5-4-1-7-8-3-2-6 had the lowest total earliness and tardiness so $\sigma$ is updated to this sequence.

To improve the solution generated by the early/ tardy-based greedy algorithm, an insertion-based local search is used. The local search generates $n$ positions at random (without repetition), and for each position the job occupying this position is removed and inserted in all possible positions.

This variable greedy algorithm is referred to as VGA in this paper. The algorithm starts with a solution developed by using the IS procedure described in subsection 2.1.1 and performs the early/tardy-based greedy algorithm with $k=1$ and the insertion-based local search. If a better solution is not found, $k$ is increased by 1 and the two steps (early/tardy-based greedy algorithm and local search) are repeated. This continues until a better solution is found or $k=n$. If a better solution is found, $k$ is set equal to 1 . If $k=n$, then a random solution is generated and $k$ is set to 1 . There are two stopping criteria for the procedure: (1) if a solution is found with zero total earliness and tardiness, the procedure stops or (2) if a time limit is exceeded, the procedure stops. Since it is possible that a solution with zero total tardiness could be found, the first check is included to stop the procedure in such a case. However, it is very unlikely that this will occur, unless the problem has very few jobs. Therefore, for almost all problems the procedure is terminated by the second check.

A second version of the variable greedy algorithm is also proposed. This algorithm, referred to as VGAB in this paper, differs from the VGA procedure by including the batch insertion neighbourhood search (BI) used in the BNS neighbourhood search in addition to the insertion-based local search after the early/tardy-based greedy algorithm is performed.

Step 1 of the early/tardy-based greedy algorithm requires $\mathrm{O}(k \ln (k))$ time. In step $2.1 \mathrm{O}(n)$ partial sequences are evaluated and each partial sequence requires $\mathrm{O}(n)$ time to evaluate, so this step requires $\mathrm{O}\left(n^{2}\right)$ time. In step $2.2 \mathrm{O}(n)$ sequences are evaluated with each sequence evaluation requiring $\mathrm{O}(n)$ time; therefore, this step requires $\mathrm{O}\left(n^{2}\right)$ time and the complexity of the early/tardy-based greedy algorithm is $\mathrm{O}\left(n^{2}\right)$.

\subsection{Genetic algorithms based on Vallada and Ruiz's (2010) genetic algorithms}

A genetic algorithm is a meta-heuristic search procedure that uses a multiple solution search technique. This approach has been found to quickly generate good solutions for a wide variety of scheduling problems. In a genetic algorithm, an initial population of chromosomes is first created, and then successive populations (or generations) of chromosomes are created using some methodology until a stopping condition is met. A chromosome corresponds to a solution for the problem. For this problem, solutions can be represented by a permutation sequence of the jobs. The $j$ th gene in a chromosome corresponds to the job in the $j$ th position of a sequence.

Vallada and Ruiz (2010) proposed three genetic algorithms for minimizing total tardiness in permutation flow shops and performed computational tests for these algorithms and other metaheuristics. They found that the genetic algorithm that used a crossover operator to generate individuals, called offspring, from two selected progenitors performed the best. Elements of this algorithm include initialization of the population, a selection mechanism, a crossover operator, mutation operator, generational scheme, local search, a restart mechanism based on the diversity of the population and the stopping condition. These elements are described in the following subsection.

2.3.1. Parameters for the genetic algorithms. In each algorithm an initial population of chromosomes (permutation sequences) is created. Two methods for creating chromosomes are used. The first method is to randomly generate chromosomes. Each chromosome (sequence) is created by first generating a random number between 0 and 1 for each job and then sorting the numbers corresponding to each job (lowest to highest) to create the sequence of jobs (chromosome). In the second method chromosomes are created by using a heuristic procedure. This method is used to seed the population with some good individuals. The proposed algorithm generates one chromosome using the earliest due date (EDD) dispatching rule and one chromosome using the $N E H_{\text {edd }}$ heuristic (Kim, 1993) and the remaining chromosomes are generated randomly.

A selection operator called $n$-tournament is used in these algorithms. With this approach a percentage of individuals, a parameter called 'pressure', is selected and the individual with the lowest total earliness and tardiness among these individuals is selected for the mating process.

The mating operator used is one point crossover. The crossover operator is applied with a $P_{c}$ probability. Two individuals are generated, called offspring, from two progenitors (chromosomes) that were selected using the $n$-tournament selection operator. First, a crossover point is randomly generated using a uniform integer distribution $[1, n]$. Then, each offspring directly inherits all the jobs from one of the parents up to the randomly generated crossover point. The last step is to copy the jobs that are missing from each offspring from the other parent in the same relative order as in the parent. For example, if the 
crossover point is three and the sequences associated with the two parents are:

Parent 1 4-5-2-3-1-6

Parent 2 1-2-3-6-5-4

The resulting children sequences are:

Child 1 4-5-2-1-3-6

Child 2 1-2-3-4-5-6

A shift mutation operator is used. With this operator, each job in a permutation sequence is extracted with a $P_{m}$ probability and is inserted in a randomly selected different position in the sequence.

Two local searches are used in the genetic algorithms. The first local search is the job insertion search used in the neighbourhood search procedures. This search is referred to as JI. In this search, a job is removed from the sequence and is inserted into all $n$ positions. The final position of the job is the position that results in the lowest total earliness and tardiness. This search is carried out for all $n$ jobs of each generated offspring after the mating and mutation operators have been applied. The JI search is also applied to the best individual found in the initial generation of the population. All of the genetic algorithms proposed in this research use the JI local search. The second local search is the batch insertion (BI) search used in the BNS neighbourhood search. Each batch is removed from the sequence of batches and then $n_{\beta}$ batch sequences are created by inserting the batch into each possible position while maintaining the relative order of the other batches. The final position of the batch is the position that results in the lowest total earliness and tardiness. If this search is incorporated in a genetic algorithm it is carried out after the JI local search has been performed. The local searches are applied with a $P_{L S}$ probability.

There are two criteria for accepting generated offspring into the population: (1) an offspring must be better (have a lower total earliness and tardiness) than the worst individual in the population and (2) the offspring has a unique sequence (there are no other individuals in the population with the same sequence).

A diversity measure is used to determine if a restart mechanism should be initiated for the algorithm. If the individuals in a population become too similar the algorithm may fail to evolve to better solutions. To avoid this condition the population is reinitialized by randomly generating new individuals with the exception of the individuals created in the initial population with heuristics. This occurs when the diversity measure falls below a certain value. See Vallada and Ruiz (2010) for a description of the diversity measure.

Two stopping criteria are used: (1) if a solution is found with zero total earliness and tardiness the procedure stops and (2) if the time limit is exceeded when a generation is completed the procedure stops. As with the Variable Greedy algorithm, the first check is unlikely to be
Table 3 Parameters for the genetic algorithms

\begin{tabular}{lc}
\hline Population size & 30 \\
Pressure & $30 \%$ \\
Crossover probability $\left(P_{c}\right)$ & $30 \%$ \\
Mutation probability $\left(P_{m}\right)$ & $2 \%$ \\
Local search probability $\left(P_{L S}\right)$ & $15 \%$ \\
\hline
\end{tabular}

successful. Therefore, and for almost all problems, it is the second check that will terminate this procedure.

In order to create offspring, the selection, crossover and mutation operators each require $\mathrm{O}(n)$ time. The JI and $\mathrm{BI}$ local searches require $\mathrm{O}\left(n^{3}\right)$ time (see analysis for these procedures in subsections 2.1.2 and 2.1.3).

Vallada and Ruiz (2010) conducted a statistical experiment to select the values for the genetic algorithm to minimize total tardiness. After doing some preliminary computational experiments we found that the parameters used by Vallada and Ruiz (2010) are also effective for minimizing total earliness and tardiness with family setups. The values for the parameters are shown in Table 3.

2.3.2. Genetic algorithms. Two versions of the genetic algorithm are proposed. The procedure referred to as GADV is Vallada and Ruiz's (2010) genetic algorithm that uses the one point crossover mating operator but is modified to incorporate family setups and the total earliness and tardiness objective. The GADVB procedure is the same as the GADV procedure except that both the $\mathrm{JI}$ and BI local searches are used.

\section{Computational tests of the procedures}

\subsection{Data and performance measures}

The procedures described in the previous section were tested on problems of various sizes in terms of the number of jobs, number of families and jobs within each family, and number of machines, for six sets of distributions of due date range and tightness, and three sets of distributions for family setup times. Each problem set consists of 10 problems. The problems within a problem set have the same number of jobs, number of families, number of jobs per family, number of machines, family setup times are drawn from the same distribution, and the due dates for the jobs are generated using the same distribution.

Eleven levels of number of jobs $(n)$ were included in the test. Some levels of job number had two levels of number of families $(F)$ and jobs per family $\left(n_{f}\right)$. This resulted in 18 combinations which are shown in Table 4.

Three levels of number of machines were tested: $m=5$, $m=10$ and $m=15$. 
Table 4 Combinations of number of jobs and families tested

\begin{tabular}{lccc}
\hline Combination & $\begin{array}{c}\text { Number of } \\
\text { jobs }(n)\end{array}$ & $\begin{array}{c}\text { Number of } \\
\text { families }(F)\end{array}$ & $\begin{array}{c}\text { Jobs per } \\
\text { family }\left(n_{f}\right)\end{array}$ \\
\hline 1 & 8 & 2 & 4 \\
2 & 10 & 2 & 5 \\
3 & 12 & 2 & 6 \\
4 & 12 & 3 & 4 \\
5 & 15 & 3 & 5 \\
6 & 20 & 2 & 10 \\
7 & 20 & 4 & 5 \\
8 & 25 & 5 & 5 \\
9 & 30 & 3 & 10 \\
10 & 30 & 5 & 6 \\
11 & 40 & 4 & 10 \\
12 & 40 & 8 & 5 \\
13 & 50 & 5 & 10 \\
14 & 50 & 10 & 5 \\
15 & 75 & 5 & 15 \\
16 & 75 & 15 & 5 \\
17 & 100 & 5 & 20 \\
18 & 100 & 10 & 10 \\
\hline
\end{tabular}

The processing times of the jobs for each machine were generated using a uniform distribution over the integers 1 and 100 . The setup times on each machine for each family were randomly generated using a uniform distribution. Three setup distributions were used. The first setup distribution (1) generated setups over the integers 1 and 50 , the second (2), over the integers 1 and 100, and the third (3), over the integers 1 and 200. The due dates for the jobs were also randomly generated using a uniform distribution over the integers $M S(1-r-R / 2)$ and $M S(1-r+R / 2)$, where $M S$ is an estimated minimum makespan found for the problem using a heuristic developed by Schaller (2000), and $R$ and $r$ are two parameters called due date range and tardiness factors. Six sets of these parameters were used for each $n, F, n_{f}$, and $m$ combination and are shown in Table 5 .

The levels of the test factors result in $(18 \times 3 \times 6 \times 3)$ 972 factor combinations in the test. Thus, a total of $(972 \times 10) 9720$ problems were considered. The procedures were coded in Turbo Pascal and were tested on a Dell Inspiron $15251.6 \mathrm{GHz}$ laptop computer for all of the problem sets.

For small-sized problems ( $n=8,10$ and 12) a branch and bound algorithm was used to obtain the optimal total earliness and tardiness. The measures of performance used to evaluate the procedures for the test problems are $\mathrm{CPU}$ time required to generate a solution, the percentage error $(\%$ Error) of the total earliness and tardiness of the solution generated by each procedure compared with the optimal total earliness and tardiness for the problem and the number of optimal solutions generated by each procedure for the small-sized problems, and the percentage difference ( $\%$ Diff) of the total earliness and tardiness of
Table 5 Due date tardiness and range factors

\begin{tabular}{lll}
\hline Set & $R$ & $r$ \\
\hline 1 & 0.5 & 0.00 \\
2 & 1.0 & 0.00 \\
3 & 0.5 & 0.25 \\
4 & 1.0 & 0.25 \\
5 & 0.5 & 0.50 \\
6 & 1.0 & 0.50 \\
\hline
\end{tabular}

the solution generated by each procedure compared with the total earliness and tardiness of the solution generated by NS procedure for the large-sized problems. The percentage error calculates the percentage of the total earliness and tardiness of the solution obtained by the heuristic procedure is greater than the optimal total earliness and tardiness. \% Error $=\left[\left(Z_{h}-Z_{O}\right) / Z_{O}\right] \times 100$, where $Z_{O}=$ the optimal total earliness and tardiness for the problem, and $Z_{h}=$ the total earliness and tardiness of the solutions generated by the heuristic procedures. $\%$ Diff $=\left[\left(Z_{h}-Z_{N S}\right) / Z_{N S}\right] \times 100$, where $Z_{N S}=$ the total earliness and tardiness for the problem obtained by the $N S$ procedure, and $Z_{h}=$ the total earliness and tardiness of the solutions generated by the other heuristic procedures.

\subsection{Results of the test}

Table 6 shows the average percentage the total earliness and tardiness of each procedure's solutions are greater than the total earliness and tardiness of the optimal solution for each of the small problem sizes $(n=8,10$ and 12) and the number of times each procedure generated an optimal solution for each problem size.

The two versions of the variable greedy algorithm performed best for these problem sizes and had average per cent errors of less than one-tenth of $1 \%$ for each of these problem sizes. The variable greedy algorithms also found the most optimal solutions. Both of the variable greedy algorithms found optimal solutions for over $95 \%$ of the problems on each of these problem sizes. The two versions of the Genetic algorithm also performed very well. The GADV procedure had average per cent errors of less than $0.2 \%$ for each of these problem sizes and solved over $95 \%$ of the problems optimally, and the GADVB procedure had average per cent errors of less than $0.4 \%$, and solved over $93 \%$ of the problems optimally. The two neighbourhood search procedures found optimal solutions for less than $75 \%$ of the problems for each problem size and had per cent errors between 1 and 5\% for each problem size.

One of the reasons the variable greedy and genetic algorithms performed better for the small problem sizes is that they used more time to generate solutions. Table 7 shows the average number of seconds per problem each 
Table 6 Average \% error from the optimal total earliness and tardiness and number of optimal solutions (in parentheses)

\begin{tabular}{|c|c|c|c|c|c|c|c|c|}
\hline \multicolumn{3}{|c|}{ Problem size } & \multicolumn{6}{|c|}{ Procedure } \\
\hline$N$ & $F / n_{f}$ & $m$ & $N S$ & $B N S$ & $G A D V$ & $G A D V B$ & $V G A$ & $V G A B$ \\
\hline 8 & $2 / 4$ & 5 & 3.39 (122) & 2.78 (127) & $0.00(180)$ & $0.02(178)$ & $0.00(180)$ & $0.00(180)$ \\
\hline 8 & $2 / 4$ & 10 & $2.08(132)$ & $1.90(130)$ & 0.00 (179) & 0.00 (179) & $0.00(180)$ & $0.00(180)$ \\
\hline 8 & $2 / 4$ & 15 & $2.54(121)$ & 1.77 (130) & $0.00(180)$ & $0.00(179)$ & $0.00(180)$ & $0.00(180)$ \\
\hline 10 & $2 / 5$ & 5 & $3.19(105)$ & $2.63(108)$ & $0.01(179)$ & 0.19 (175) & 0.01 (179) & $0.01(177)$ \\
\hline 10 & $2 / 5$ & 10 & $2.32(114)$ & 1.94 (119) & 0.03 (177) & $0.08(166)$ & $0.01(179)$ & 0.00 (179) \\
\hline 10 & $2 / 5$ & 15 & $2.28(112)$ & $2.22(109)$ & $0.00(178)$ & $0.10(170)$ & $0.00(180)$ & $0.00(180)$ \\
\hline 12 & $2 / 6$ & 5 & $3.93(92)$ & $3.60(91)$ & $0.06(176)$ & $0.22(166)$ & $0.02(175)$ & 0.02 (176) \\
\hline 12 & $2 / 6$ & 10 & $4.42(76)$ & $4.13(86)$ & $0.15(170)$ & $0.36(155)$ & $0.05(173)$ & $0.06(174)$ \\
\hline 12 & $2 / 6$ & 15 & $3.46(72)$ & $3.26(73)$ & 0.15 (174) & $0.25(159)$ & 0.02 (179) & 0.02 (177) \\
\hline 12 & $3 / 4$ & 5 & $4.32(87)$ & $3.00(92)$ & $0.14(169)$ & 0.08 (168) & 0.01 (179) & $0.00(179)$ \\
\hline 12 & $3 / 4$ & 10 & $3.53(86)$ & $2.55(94)$ & $0.06(176)$ & $0.13(164)$ & 0.01 (177) & $0.03(175)$ \\
\hline 12 & $3 / 4$ & 15 & $3.58(57)$ & $3.32(63)$ & 0.07 (172) & $0.12(166)$ & 0.03 (177) & $0.03(178)$ \\
\hline
\end{tabular}

Table 7 Average seconds per problem for $m=15$

\begin{tabular}{|c|c|c|c|c|c|c|c|}
\hline \multicolumn{2}{|c|}{ Problem size } & \multicolumn{6}{|c|}{ Procedure } \\
\hline$N$ & $F / n_{f}$ & $N S$ & $B N S$ & $G A D V$ & $G A D V B$ & $V G A$ & $V G A B$ \\
\hline 8 & $2 / 4$ & 0.02 & 0.03 & 7.53 & 7.53 & 7.53 & 7.53 \\
\hline 10 & $2 / 5$ & 0.05 & 0.06 & 9.40 & 9.40 & 9.39 & 9.39 \\
\hline 12 & $2 / 6$ & 0.09 & 0.11 & 11.3 & 11.3 & 11.3 & 11.3 \\
\hline 12 & $3 / 4$ & 0.10 & 0.13 & 11.3 & 11.3 & 11.3 & 11.3 \\
\hline 15 & $3 / 5$ & 0.23 & 0.26 & 14.2 & 14.2 & 14.1 & 14.1 \\
\hline 20 & $2 / 10$ & 0.73 & 0.69 & 18.9 & 18.9 & 18.8 & 18.8 \\
\hline 20 & $4 / 5$ & 0.83 & 0.88 & 18.9 & 19.0 & 18.8 & 18.8 \\
\hline 25 & $5 / 5$ & 2.06 & 2.00 & 23.7 & 24.1 & 23.5 & 23.5 \\
\hline 30 & $3 / 10$ & 3.98 & 3.68 & 28.6 & 28.9 & 28.2 & 28.2 \\
\hline 30 & $5 / 6$ & 4.25 & 3.96 & 28.7 & 29.1 & 28.2 & 28.3 \\
\hline 40 & $4 / 10$ & 14.1 & 11.9 & 38.5 & 39.2 & 37.7 & 37.7 \\
\hline 40 & $8 / 5$ & 16.1 & 13.5 & 38.6 & 41.0 & 37.7 & 37.7 \\
\hline 50 & $5 / 10$ & 33.3 & 27.3 & 49.1 & 50.7 & 47.3 & 47.3 \\
\hline 50 & $10 / 5$ & 37.5 & 30.7 & 49.1 & 54.5 & 47.3 & 47.4 \\
\hline 75 & $5 / 15$ & 73.8 & 73.7 & 77.5 & 82.5 & 71.5 & 71.6 \\
\hline 75 & $15 / 5$ & 74.3 & 74.1 & 78.7 & 105.8 & 71.6 & 72.0 \\
\hline 100 & $5 / 20$ & 101.6 & 101.3 & 109.8 & 116.4 & 96.4 & 96.7 \\
\hline 100 & $10 / 10$ & 101.1 & 100.9 & 110.2 & 143.1 & 96.3 & 97.2 \\
\hline
\end{tabular}

procedure used to generate a solution for each problem size when $m=15$.

Since the time required by each of the procedures to generate solutions was relatively linear with respect to the number of machines the results for $m=5$ and 10 are omitted.

The variable greedy and genetic algorithms continue until the time limit criteria for stopping is reached whereas the neighbourhood searches terminate much more quickly on the small problem sizes. The neighbourhood searches usually terminate before the time limit stopping criteria is reached for problem sizes consisting of 50 jobs or less.

Table 8 shows the Average \% Diff measure for each level of $n$ for problems with 15 or more jobs.
Table 8 Average \% Diff from NS solution by number of jobs

\begin{tabular}{lrrrrr}
\hline Number of jobs (n) & \multicolumn{5}{c}{ Procedure } \\
\cline { 2 - 6 } & BNS & GADV & GADVB & VGA & VGAB \\
\hline 15 & -1.06 & -4.62 & -4.54 & -4.69 & -4.65 \\
20 & -0.72 & -4.92 & -4.65 & -4.48 & -4.21 \\
25 & -1.92 & -5.95 & -5.98 & -3.99 & -4.22 \\
30 & -1.84 & -5.57 & -5.71 & -2.22 & -2.26 \\
40 & -3.19 & -5.17 & -6.01 & 0.52 & 0.12 \\
50 & -4.02 & -4.51 & -5.92 & 3.18 & 2.31 \\
75 & -8.49 & -8.85 & -10.79 & -2.41 & -3.91 \\
100 & -9.12 & -11.87 & -14.46 & -7.23 & -8.55 \\
\hline
\end{tabular}

Figure 1 plots these results. For problem sizes up to 20 jobs the genetic and variable greedy algorithms perform best and are relatively close. When $n>20$, the genetic algorithms performed the best. As $n$ increases the relative performance of the GADVB procedure improves and is the best when $n \geqslant 25$. When $n=75$ or 100 the $\%$ Diff for the GADVB procedure was greater than $10 \%$. Although the variable greedy algorithms are best when $n$ is low their performance declines rapidly as $n$ initially is increased and for $n=40$ and 50 were worse than even the NS procedure.

The use of a batch neighbourhood search proved to be beneficial as problem size increased. The BNS procedure outperformed the NS procedure for all problem sizes. The GADVB procedure outperformed the GADV procedure when $n \geqslant 25$ and the VGAB procedure also outperformed the VGA procedure when $n \geqslant 25$.

Table 9 shows the Average \% Diff measure for each level of number of machines $(m)$.

Figure 2 plots these results. The number of machines did not appear to affect the relative quality of the solutions generated by the procedures as the average \% Diff's of the procedures are consistent across the three levels 


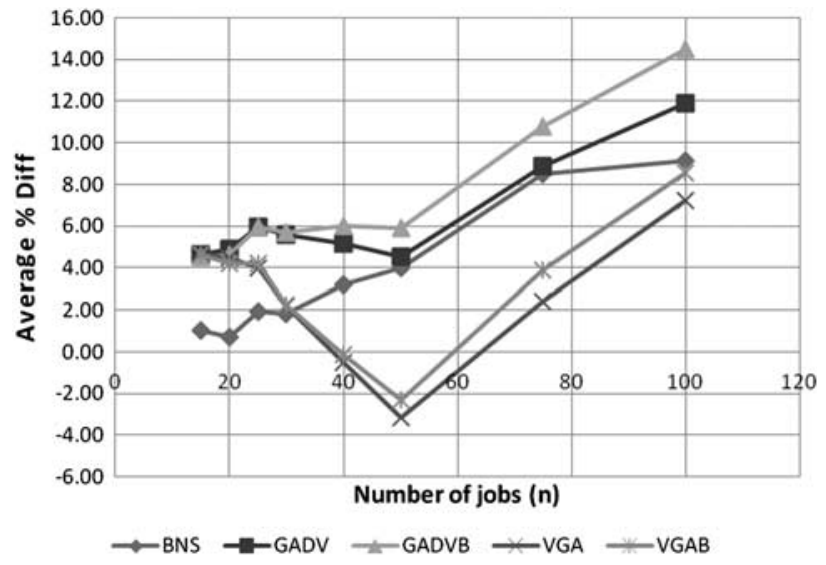

Figure 1 Average \% Diff by number of jobs $(n)$.

Table 9 Average \% Diff from NS solution by number of machines

\begin{tabular}{|c|c|c|c|c|c|}
\hline \multirow[t]{2}{*}{ Number of machines $(m)$} & \multicolumn{5}{|c|}{ Procedure } \\
\hline & $B N S$ & $G A D V$ & $G A D V B$ & $V G A$ & $V G A B$ \\
\hline 5 & -4.27 & -6.34 & -7.36 & -2.06 & -3.00 \\
\hline 10 & -4.15 & -6.74 & -7.67 & -2.51 & -2.86 \\
\hline 15 & -3.94 & -6.72 & -7.59 & -2.71 & -3.12 \\
\hline
\end{tabular}

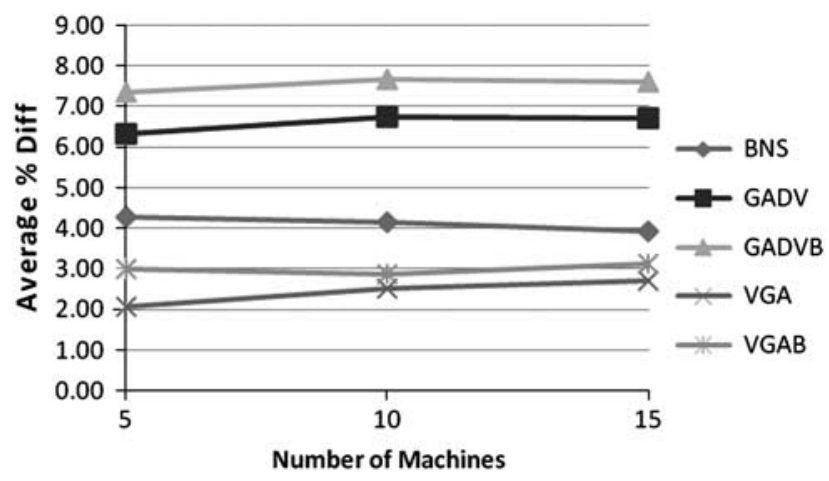

Figure 2 Average \% Diff by number of machines.

of machines. For each level of $m$ the ranking of the procedures is: (1) GADVB, (2) GADV, (3) BNS, (4) VGAB, and (5) VGA. These results also show the advantage of including batch neighbourhood searches as the procedures that included a batch neighbourhood search outperformed the corresponding procedure that did not.

Table 10 shows the Average \% Diff measure for each of the due date tightness and range factors.

Figure 3 plots the results for the Average \% Diff measure by due date tightness factor and Figure 4 by due date range factor. These results also show that the genetic algorithms performed best followed by the BNS procedure.
Table 10 Average \% Diff from NS solution by due date tightness and range factors.

\begin{tabular}{|c|c|c|c|c|c|c|}
\hline \multicolumn{2}{|c|}{$\begin{array}{l}\text { Due date } \\
\text { tightness and } \\
\text { range factors }\end{array}$} & \multicolumn{5}{|c|}{ Procedure } \\
\hline$r$ & $R$ & $B N S$ & $G A D V$ & $G A D V B$ & $V G A$ & $V G A B$ \\
\hline 0.00 & 0.50 & -2.14 & -4.23 & -4.21 & 0.11 & 0.08 \\
\hline 0.00 & 1.00 & -1.99 & -6.26 & -5.92 & -1.16 & -1.28 \\
\hline 0.25 & 0.50 & -4.64 & -7.80 & -8.62 & -3.92 & -4.31 \\
\hline 0.25 & 1.00 & -5.33 & -9.19 & -9.78 & -4.55 & -5.24 \\
\hline 0.50 & 0.50 & -5.25 & -6.14 & -8.48 & -2.72 & -3.44 \\
\hline 0.50 & 1.00 & -5.38 & -5.96 & -8.24 & -2.32 & -3.77 \\
\hline
\end{tabular}

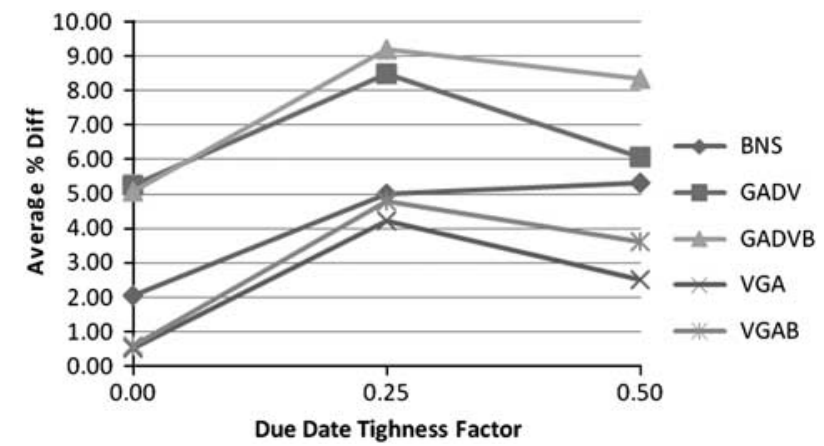

Figure 3 Average \% Diff by due date tightness factor $(r)$.

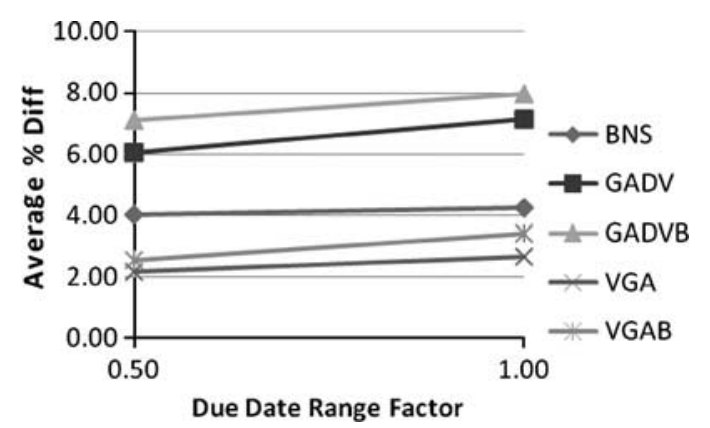

Figure 4 Average \% Diff by due date range factor $(R)$.

The performance of the procedures compared with the $N S$ procedure improves when $R=1.00$ and when $r>0.00$.

Table 11 shows the Average \% Diff measure for each setup distribution level.

Figure 5 plots these results. These results also show that the genetic algorithms perform best. It can also be seen that including a batch neighbourhood search becomes more beneficial as the size of setups are increased as the relative performance of procedures with a batch neighbourhood search improves compared with their counterparts without a batch neighbourhood search.

To summarize the results the genetic and variable greedy algorithms generate the best solutions for small-sized 
Table 11 Average \% Diff from NS solution by setup distribution

\begin{tabular}{lccccc}
\hline Setup & \multicolumn{5}{c}{ Procedure } \\
\hline Distribution & BNS & GADV & GADVB & VGA & VGAB \\
\hline $1(1-50)$ & -2.58 & -6.33 & -6.12 & -3.26 & -3.24 \\
$2(1-100)$ & -4.09 & -6.86 & -7.47 & -2.64 & -3.07 \\
$3(1-200)$ & -5.69 & -6.60 & -9.04 & -1.38 & -2.66 \\
\hline
\end{tabular}

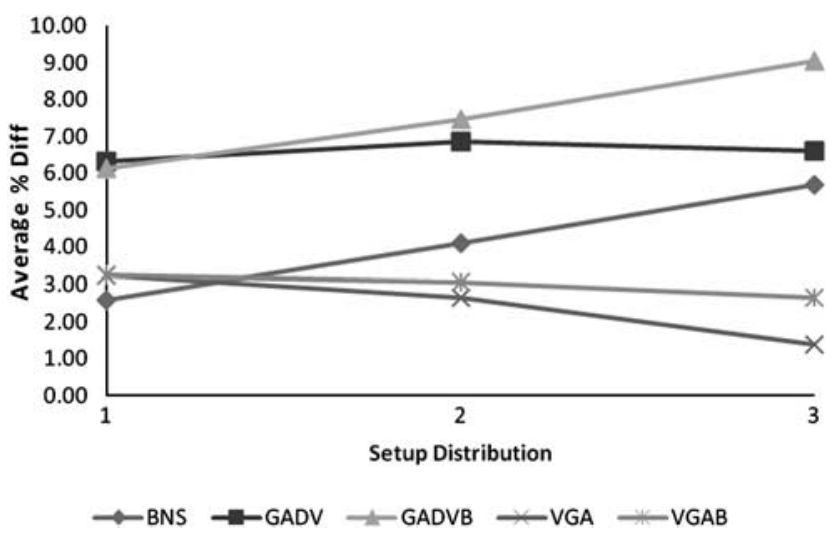

Figure 5 Average \% Diff by setup distribution.

problems but as the number of jobs increases the GADVB procedure generates the best solutions. The GADVB procedure was also consistently best over all levels of number of machines and setup distributions and the genetic algorithms were best for all the due date tightness and range factors. Procedures with a batch neighbourhood search consistently outperformed the corresponding procedure that did not include a batch neighbourhood search.

\section{Impact of a reduction in setup times on total earliness and tardiness}

It is commonly recognized that the existence of long setup times is a negative factor in production operations. Long setup times are associated with increased costs for performing the setups, and since production equipment is not producing during the setup, long setup times also reduce production capacity, which represents an opportunity cost. To help offset the cost and reduction of production capacity caused by long family setups, jobs belonging to the same setup families are batched and produced together, thereby reducing the total number of setups and the time spent setting up. This batching of jobs makes it difficult to match the production of individual jobs with their due dates. Some jobs in a batch are produced before their due dates and have to be held in inventory which is a form of waste. Also these early jobs are using production capacity that could be used to produce jobs that have current due dates and hence are delayed so that they become tardy. This is another form of waste. Shingo (1985) developed a set of principles called Single Minute Exchange of Die (SMED) to reduce setup times. These principles are relatively easy to implement and have been widely utilized to reduce setup times. Setup time reductions of $50-75 \%$ are very common utilizing this approach. This section describes a test to help determine how reducing setup times using methods such as SMED in permutation flow shops affects the total earliness and tardiness incurred.

The data for the test were created in a fashion similar to that described in Section 3.1. Problems were created for the problem size parameters: $n=50, F=5, n_{f}=10$ for all $f=1$ to $F, m=10$; and $n=50, F=10, n_{f}=5$ for all $f=1$ to $F$, and $m=10$. Each problem set consisted of 10 problems. Setup times for each family on each machine were generated in two steps. In the first step a number is generated using a uniform distribution over the integers 1 and 50. Then in the second step the number generated is multiplied by four to obtain the setup time. Processing times and due dates were randomly generated using the approach described in Section 3.1. For each problem that was created, two additional problems were created. Both of the additional problems consisted of the same data as the original problem with the exception of the setup times. One problem was created by dividing the setup times by two (a $50 \%$ reduction) and the other problem was created by dividing the setup times by four (a 75\% reduction).

Since the GADVB procedure performed the best for the problems with 50 jobs $(n=50)$, it was used to generate a solution for each problem and the resulting total earliness and tardiness, makespan and the number of setups were recorded. Table 12 shows results for each problem size.

The table shows the per cent reduction in total earliness and tardiness and makespan and the per cent increase in the total number of setups when the setup times were reduced 50 and $75 \%$. The results show that there were large decreases in total earliness and tardiness and large increases in the number of setups for each problem size when there was a $50 \%$ reduction in setup times. When the reduction in setup times was $75 \%$ there was a further reduction in total earliness and tardiness and the increase in the number of setups was significant even when compared with those of the $50 \%$ reduction in setup times. The makespan was decreased with the $50 \%$ reduction in setup times and was further decreased with the $75 \%$ reduction in setup times but not as significantly as the decrease in total earliness and tardiness. The reason why the makespans did not decrease by a larger amount can be attributed to the increase in the number of setups which offsets some of the reduction in setup times.

The due date tightness and range factors affects these results. To illustrate how these factors affect total earliness 
Table 12 Impact of reduction in setup times for each problem size $(n=50$ and $m=10)$

\begin{tabular}{|c|c|c|c|c|c|c|c|}
\hline \multicolumn{2}{|c|}{ Problem size } & \multicolumn{3}{|c|}{$50 \%$ reduction in setup times } & \multicolumn{3}{|c|}{$75 \%$ reduction in setup times } \\
\hline \multirow[b]{2}{*}{$F$} & \multirow[b]{2}{*}{$n_{f}$} & \multicolumn{2}{|c|}{$\%$ reduction } & \multirow{2}{*}{$\begin{array}{c}\% \text { increase } \\
\text { Setups }\end{array}$} & \multicolumn{2}{|c|}{$\%$ reduction } & \multirow{2}{*}{$\begin{array}{c}\% \text { increase } \\
\text { Setups }\end{array}$} \\
\hline & & Total $E / T$ & Makespan & & Total $E / T$ & Makespan & \\
\hline 5 & 10 & 25.19 & 6.73 & 91.12 & 31.34 & 10.24 & 182.89 \\
\hline 10 & 5 & 32.59 & 10.86 & 36.36 & 42.44 & 16.35 & 83.57 \\
\hline
\end{tabular}

Table 13 Impact of reduction in setup times by the due date tightness and range factors for $F=10, n_{f}=5$ and $m=10$

\begin{tabular}{|c|c|c|c|c|c|c|c|}
\hline \multicolumn{2}{|c|}{ Due date tightness and range factors } & \multicolumn{3}{|c|}{$50 \%$ reduction in setup times } & \multicolumn{3}{|c|}{$75 \%$ reduction in setup times } \\
\hline \multirow[b]{2}{*}{$r$} & \multirow[b]{2}{*}{$R$} & \multicolumn{2}{|c|}{$\%$ reduction } & \multirow{2}{*}{$\frac{\% \text { increase }}{\text { Setups }}$} & \multicolumn{2}{|c|}{$\%$ reduction } & \multirow{2}{*}{$\frac{\% \text { increase }}{\text { Setups }}$} \\
\hline & & Total $E / T$ & Makespan & & Total $E / T$ & Makespan & \\
\hline 0.00 & 0.50 & 16.17 & 10.00 & 64.58 & 12.55 & 15.12 & 115.63 \\
\hline 0.00 & 1.00 & 22.88 & 8.04 & 57.05 & -6.53 & 12.74 & 54.36 \\
\hline 0.25 & 0.50 & 33.99 & 10.59 & 21.43 & 47.34 & 15.74 & 122.45 \\
\hline 0.25 & 1.00 & 53.24 & 9.97 & 11.94 & 75.83 & 15.67 & 32.09 \\
\hline 0.50 & 0.50 & 30.43 & 13.15 & 20.59 & 46.89 & 18.99 & 89.71 \\
\hline 0.50 & 1.00 & 37.67 & 14.40 & 36.59 & 58.18 & 21.03 & 131.71 \\
\hline Total & & 32.59 & 10.86 & 36.36 & 42.44 & 16.35 & 83.57 \\
\hline
\end{tabular}

and tardiness, makespan and the number of setups as setup time is reduced, Table 13 shows the results for each set of due date range and tightness factors for the $F=10, n_{f}=5$ and $m=10$ problem size.

The results in this table show the due date tightness and range factors affect the results in terms of total earliness and tardiness obtained when setup times are reduced. When the due date tightness factor is very low $(r=0.00)$ the reduction in total earliness and tardiness when setup times are reduced by $50 \%$ is considerably less than when the due date tightness factor is set at higher levels $(r=0.25$ or 0.50$)$. When $r=0.00$ and setup times are reduced by $75 \%$ the total earliness and tardiness actually increases compared with the $50 \%$ setup reduction and in the case of $r=0.00$ and $R=1.00$ the total earliness and tardiness is higher than when there is no reduction in setup times. When the due date tightness factor is set at 0.25 or 0.50 the reduction in total earliness and tardiness is very high with a $50 \%$ reduction in setup times and is substantially higher when the reduction in setup times is $75 \%$. Also, the reduction in total earliness and tardiness is greater when $R=1.00$. The restriction that only non-delay schedules are considered is an important factor contributing to these results. When due dates are loose and there is excess capacity then as setup times are reduced there is even greater excess capacity and several jobs are completed earlier even if additional setups are used causing the early completion penalty to increase. It would be interesting to see how removing the restriction that only non-delay schedules are considered affects total earliness and tardiness when setup times are reduced. The results of the current test show that when capacity is reasonably matched with the demand, a reduction in setup times allows for a better matching of production of jobs with their due dates enabling a much lower total earliness and tardiness to be achieved.

\section{Conclusion}

In this paper several heuristics that were based on procedures found to be effective for minimizing total tardiness in permutation flow shops were proposed for minimizing total earliness and tardiness for permutation flow shops with family setups. These heuristics included neighbourhood searches, variable greedy algorithms and genetic algorithms.

The proposed procedures were tested on problems of various sizes in terms of the number of jobs, number of families included, and the number of machines. Also considered were three distributions of family setups and six sets of distributions that determine the tightness and range of due dates. The solutions generated were compared against optimal solutions for small-sized problems and the solutions found by one of the procedures for large-sized problems.

The results of the tests showed that the variable greedy algorithms were very effective for the small-sized problems. 
The best performing procedure for larger sized problems was a genetic algorithm (GADVB) that included a neighbourhood of batch sequences in addition to a neighbourhood search for job sequences. Also, incorporating a batch sequence local search into the various algorithms improved their performance.

A test was also conducted to see the effect of reducing setup times on the total earliness and tardiness obtained in scheduling. It was found that if setup times are reduced, then total earliness and tardiness can be significantly reduced. The reduction in total earliness and tardiness is achieved not only from additional effective capacity obtained by reducing setup times, but also utilizing the resources more effectively by scheduling smaller batches of jobs belonging to each family so that production of individual jobs is better matched to their due dates. The test also showed that the tightness of the due dates affected the reduction in total earliness and tardiness when setup times are reduced. When there was excess capacity and due dates were not tight reducing setup times did not impact earliness and tardiness as much as when capacity was tighter. This could be due in large part to the restriction that only non-delay schedules were considered. It would be interesting to approach the problem where non-delay schedules are considered and the use of unforced inserted idle time is permitted. This would be a productive area for future work.

\section{References}

Baker KR and Scudder GD (1990). Sequencing with earliness and tardiness penalties: A review. Operations Research 38(1): 22-36.

Chandra P, Mehta P and Tirupati D (2009). Permutation flow shop scheduling with earliness and tardiness penalties. International Journal of Production Research 47(20): 5591-5610.

Cheng TCE, Gupta JND and Wang G (2000). A review of flowshop scheduling research with setup times. Production and Operations Management 9(3): 262-282.

Framinan JM and Leisten R (2008). Total tardiness minimization in permutation flowshops: A simple approach based on a variable greedy algorithm. International Journal of Production Research 46(22): 6479-6498.

Hasija S and Rajendran C (2004). Scheduling in flowshops to minimize total tardiness of jobs. International Journal of Production Research 42(11): 2289-2301.
Hoogeveen H (2005). Multicriteria scheduling. European Journal of Operational Research 167(3): 592-623.

Jorden C (1996). Batching and Scheduling: Models and Methods for Several Problem Classes. Springer: Berlin, Germany.

Kanet JJ and Sridharan V (2000). Scheduling with inserted idle time: Problem taxonomy and literature review. Operations Research 48(1): 99-110.

Kim Y (1993). Heuristics for flowshop scheduling problems minimizing mean tardiness. Journal of the Operational Research Society 44(1): 19-28.

Kim Y, Lim HG and Park MW (1996). Search heuristics for a flowshop scheduling problem in a printed circuit board assembly process. European Journal of the Operational Research 91(1): 124-143.

Korman K (1994). A pressing matter. Video February: 46-50.

Landis K (1993). Group technology and cellular manufacturing in the Westvaco Los Angeles VH Department. Project report in IOM 581, School of Business, University of Southern California.

Madhushini N, Rajendran C and Deepa Y (2009). Branchand-bound algorithms for scheduling in permutation flowshops to minimize the sum of weighted flowtime/sum of weighted tardiness/sum of weighted flowtime and weighted tardiness/ sum of weighted flowtime, weighted tardiness and weighted earliness of jobs. Journal of the Operational Research Society 60(7): 991-1004.

Moslehi G, Mirzaee M, Vasei M and Azaron A (2009). Twomachine flow shop scheduling to minimize the sum of maximum earliness and tardiness. International Journal of Production Economics 122(2): 763-773.

Parthasarathy S and Rajendran C (1997). A simulated annealing heuristic for scheduling to minimize mean weighted tardiness in a flowshop with sequence-dependent setup times of jobs-A case study. Production Planning and Control 8(5): 475-483.

Schaller JE (2000). A comparison of heuristics for family and job scheduling in a flow-line manufacturing cell. International Journal of Production Research 38(2): 287-308.

Shingo S (1985). SMED: A Revolution in Manufacturing. Cambridge, MA: Productivity Press.

Valente JMS (2009). Beam search heuristics for the single machine scheduling problem with linear earliness and quadratic tardiness costs. Asia-Pacific Journal of Operational Research 26(3): 319-339.

Vallada E and Ruiz R (2010). Genetic algorithms with path relinking for the minimum tardiness permutation flowshop problem. Omega 38(1/2): 57-67.

Vallada E, Ruiz R and Minella G (2008). Minimising total tardiness in the m-machine flowshop problem: A review and evaluation of heuristics and metaheuristics. Computers \& Operations Research 4(4): 1350.

Received June 2011; accepted July 2012 after two revisions 